\title{
Decomposition and Construction of Neighbourhood Operations Using Linear Algebra
}

\author{
Atsushi Imiya, Yusuke Kameda, and Naoya Ohnishi \\ Institute of Media and Information Technology, Chiba University, Japan \\ School of Science and Technology, Chiba University, Japan
}

\begin{abstract}
In this paper, we introduce a method to express a local linear operated in the neighbourhood of each point in the discrete space as a matrix transform. To derive matrix expressions, we develop a decomposition and construction method of the neighbourhood operations using algebraic properties of the noncommutative matrix ring. This expression of the transforms in image analysis clarifies analytical properties, such as the norm of the transforms. We show that the symmetry kernels for the neighbourhood operations have the symmetry matrix expressions.
\end{abstract}

\section{Introduction}

Linear vs Nonlinear, Local vs Global, Shift-Invariant vs Shift-Variant are fundamental characteristics for classifying the mathematical properties of the operations for image processing. Geometric vs Algebraic and Combinatorial vs Analytical are fundamental methodologies for analysing and designing algorithms and operations in discrete geometry and digital image processing.

In this paper, using an algebraic method for the description of the neighbourhood operations, we introduce the norm of the local operations as a classification criterion for shift-invariant local linear operations for image processing [3]. To define the norm of the operations, we introduce a decomposition and construction method for the neighbourhood operations of digital image processing using algebraic properties of the noncommutative matrix ring. Using this decomposition and construction method, we develop a method to express the neighbourhood operations [12] in matrix forms [46]7]. The matrix expressions of the neighbourhood operations yield the operator norm [13] of the operations. This norm of the neighbourhood operations allows us to deal with the neighbourhood operations analytically, that is, we can define the spectra of the neighbourhood operations.

In signal processing and analysis, it is well known that a shift-invariant linear operation is expressed as a convolution kernel. Furthermore, a linear transform in a finite dimensional space is expressed as a matrix 41312. It is also possible to express a shift-invariant operation as a band-diagonal matrix 345. However, this expression is not usually used in signal processing and analysis. In numerical computation of the partial differential equations, approximations of the partial differentiations in discrete operations are one of the central issues 
101113. The discrete approximations of the partial differentiations are called the neighbourhood operation in digital signal and image processing. In mathematical morphology, a neighbourhood operation is expressed as a small binary matrix. This small matrix, which is called the structure element in binary mathematical morphology [2], expresses the local distribution of boolean value zero and one in a small region.

For the analysis and expression of digital image transformations from the viewpoint of functional analysis, we introduce a method to describe the neighbourhood operations in the matrix form. Two kinds of expressions for the neighbourhood operations, the tensor expression [15], which is called the matrix expression in mathematical morphology [2], and the convolution operation, which expresses the operation as a convolution between input and kernel, are well established methods in digital signal and image processing. The kernel of the convolution operation is expressed as a small matrix [3] in the context of digital image processing. We address the third expression of the operations in digital image processing. The matrix expression of the linear operation derives the mathematical definition of the norm of the linear operation [410]. In digital image analysis, geometrical properties of the results of the operation are the most important concerns, since the transformations are operated to extract geometrical features such as the boundary curves, medial axis, and corners. The norm of the linear operation allows us to analyse spectral properties of the operations. The spectrum of operations determine some analytical properties of the transformation in the space of functions.

\section{Mathematical Preliminaries}

We assume that the sampled image $f(i, j, k)$ exists in the $M \times M \times M$ grid region, that is, we express $f_{i j k}$ as the value of $f(i, j, k)$ at the point $(i, j, k)^{\top}$ in three-dimensional discrete space $\mathbf{Z}^{3}$. We use the following notations to express shift-invariant local operation as three-dimensional discrete operations.

For the sequence $\boldsymbol{w}=\left(w_{(-1)}, w_{(o)}, w_{(+1)}\right)$,

$$
\boldsymbol{w} f_{i j k}=w_{(-1)} f_{i-1 j k}+w_{(0)} f_{i j k}+w_{(+1)} f_{i+1 j k} .
$$

For the $3 \times 3$ matrix $\boldsymbol{W}=\left(\boldsymbol{w}_{(-1)}, \boldsymbol{w}_{(0)}, \boldsymbol{w}_{(+1)}\right)$,

$$
\boldsymbol{W} f_{i j k}=\boldsymbol{w}_{(-1)} f_{i j-1 k}+\boldsymbol{w}_{(0)} f_{i j k}+\boldsymbol{w}_{(+1)} f_{i j+1 k} .
$$

For the $3 \times 3 \times 3$ tensor $\mathbf{W}=\left(\boldsymbol{W}_{(-1)}, \boldsymbol{W}_{(0)}, \boldsymbol{W}_{(+1)}\right)$,

$$
\mathbf{W} f_{i j k}=\boldsymbol{W}_{(-1)} f_{i j k-1}+\boldsymbol{W}_{(0)} f_{i j k}+\boldsymbol{W}_{(+1)} f_{i j k+1} \text {. }
$$

where $\boldsymbol{W}_{ \pm}$and $\boldsymbol{W}_{0}$ are $3 \times 3$ matrices in the $3 \times 3 \times 3$ tensor $\mathbf{W}$. For the three-dimensional vector function $\boldsymbol{x}_{i j k}=\boldsymbol{x}(i, j, k)=\left(x_{i j k}, y_{i j k}, z_{i j k}\right)^{\top}$ defined in $\mathbf{Z}^{3}$,

$$
\mathbf{W} \boldsymbol{x}=\left(\mathbf{W} x_{i j k}, \mathbf{W} y_{i j k}, \mathbf{W} z_{i j k}\right)^{\top}
$$


For the $3 \times 3 \times 3$ tensor $\mathbf{W}$, the $5 \times 5 \times 5$ tensor $\mathbf{W}^{2}$ is defined as

$$
\mathbf{W}^{2}=\left(\left(w_{i j k}^{(2)}\right)\right) \quad w_{i j k}^{(2)}=\sum_{p q r} w_{i-p j-q, j-r} w_{p q r} .
$$

where $\left(\left(w_{i j k}\right)\right)$ expresses the tensor whose $i j k$ element is $w_{i j k}$. These vector, matrix, and tensor are called convolution kernels for one-, two-, and three- dimensional digital functions in digital signal and image processing. For these vectors, matrices, and tensors, we define symmetry.

Definition 1. If $w_{-1}=w_{+1}, \boldsymbol{w}_{-1}=\boldsymbol{w}_{+1}$, and $\boldsymbol{W}_{-1}=\boldsymbol{W}_{+1}$, we call that vectors, matrices, and tensors are kernel symmetry.

In this paper, we derive a method to describe the kernel symmetry local operations as matrices and define the norm of operations using matrix expressions.

Hereafter, we use $\mathbf{T}$ and $\boldsymbol{T}$ to express the tensor and the matrix, respectively, of the linear transform $T$ to arrays in $n$-dimensional discrete space $\mathbf{Z}^{n}$ for $n \geq 3$.

We describe the fundamental numerical differentiations in tensor forms. In numerical differentiation 1113, since

$$
\frac{\partial}{\partial x} f(x, y, z) \cong \Delta_{i} f_{i j k}=f_{i+\frac{1}{2} j, k}-f_{i-\frac{1}{2} j k}
$$

we have

$$
\frac{\partial^{2}}{\partial x^{2}} f \cong \Delta_{i}^{2} f_{i j k}=f_{i+1 j k}-2 f_{i j k}+f_{i-1 j k}
$$

If linear approximation is used to derive $f_{i+\frac{1}{2} j k}$ from $f_{i j k}$ and $f_{i+1 j k}$, we have

$$
\boldsymbol{\Delta}_{i} f_{i j k}=f_{i+1 j, k}-f_{i-1 j k} .
$$

Furthermore, the gradient $\nabla f=\left(f_{x}, f_{y}, f_{z}\right)^{\top}$ and the Laplacian $\nabla^{2} f$ for the numerical computation of partial differential equations are approximated as

$$
\begin{aligned}
\left(\begin{array}{l}
f_{x} \\
f_{y} \\
f_{z}
\end{array}\right) & \cong\left(\begin{array}{c}
\boldsymbol{\Delta}_{i} f_{i j k} \\
\boldsymbol{\Delta}_{j} f_{i j k} \\
\boldsymbol{\Delta}_{k} f_{i j k}
\end{array}\right)=\left(\begin{array}{c}
\left(f_{i+1 j k}-f_{i j k}\right)+\left\{-\left(f_{i-1 j k}-f_{i j k}\right)\right\} \\
\left(f_{i j k+1}-f_{i j k}\right)+\left\{-\left(f_{i j k-1}-f_{i j k}\right)\right\} \\
\left(f_{i j+1 k}-f_{i j k}\right)+\left\{-\left(f_{i j-1 k}-f_{i j k}\right)\right\}
\end{array}\right) \\
& =\left(\begin{array}{c}
f_{i+1 j k}-f_{i-1 j k} \\
f_{i j+1 k}-f_{i j-1 k} \\
f_{i j k+1}-f_{i j k-1}
\end{array}\right)
\end{aligned}
$$

and

$$
\begin{aligned}
\nabla^{2} f \cong & \Delta_{i}^{2} f_{i j k}+\Delta_{j}^{2} f_{i j k}+\Delta_{k}^{2} f_{i j k} \\
= & \left(f_{i+1 j k}-2 f_{i j k}+f_{i-1 j k}\right)+\left(f_{i j+1 k}-2 f_{i j k}+f_{i j-1 k}\right) \\
& +\left(f_{i j k+1}-2 f_{i j k}+f_{i j k-1}\right) \\
= & \left(f_{i+1 j k}+f_{i-1 j k}+f_{i j+1 k}+f_{i j-1 k}+f_{i j k+1}+f_{i j k-1}\right)-6 f_{i j k},
\end{aligned}
$$

respectively. Therefore, the Laplacian for the numerical computation of the partial differential equation is six times the 6-connected Laplacian from the viewpoint of digital image analysis. 
The tensors [15] $\mathbf{A}_{6}, \mathbf{A}_{12}$, and $\mathbf{A}_{8}$ for the computation of averages in the 6-, 18-, and 26- neighbourhoods in $\mathbf{Z}^{3}$ for volumetric image analysis are

$$
\begin{aligned}
& \mathbf{A}_{6}=\frac{1}{6}\left(\left(\begin{array}{lll}
0 & 0 & 0 \\
0 & 1 & 0 \\
0 & 0 & 0
\end{array}\right)\left(\begin{array}{lll}
0 & 1 & 0 \\
1 & 0 & 1 \\
0 & 1 & 0
\end{array}\right)\left(\begin{array}{lll}
0 & 0 & 0 \\
0 & 1 & 0 \\
0 & 0 & 0
\end{array}\right)\right) \\
& \mathbf{A}_{12}=\frac{1}{12}\left(\left(\begin{array}{lll}
0 & 1 & 0 \\
1 & 0 & 1 \\
0 & 1 & 0
\end{array}\right)\left(\begin{array}{lll}
1 & 0 & 1 \\
0 & 0 & 0 \\
1 & 0 & 1
\end{array}\right)\left(\begin{array}{lll}
0 & 1 & 0 \\
1 & 0 & 1 \\
0 & 1 & 0
\end{array}\right)\right), \\
& \mathbf{A}_{8}=\frac{1}{8}\left(\left(\begin{array}{lll}
1 & 0 & 1 \\
0 & 0 & 0 \\
1 & 0 & 1
\end{array}\right)\left(\begin{array}{lll}
0 & 0 & 0 \\
0 & 0 & 0 \\
0 & 0 & 0
\end{array}\right)\left(\begin{array}{lll}
1 & 0 & 1 \\
0 & 0 & 0 \\
1 & 0 & 1
\end{array}\right)\right) .
\end{aligned}
$$

These tensors have the following properties,

$$
\mathbf{A}_{18}=\frac{6}{18} \mathbf{A}_{6}+\frac{12}{18} \mathbf{A}_{12}, \quad \mathbf{A}_{26}=\frac{6}{26} \mathbf{A}_{6}+\frac{12}{26} \mathbf{A}_{12}+\frac{8}{26} \mathbf{A}_{8} .
$$

Therefore, the tensors $\mathbf{L}_{6}, \mathbf{L}_{12}$, and $\mathbf{L}_{8}$ for the computation of Laplacians in 6-, 18-, and 26- neighbourhoods are

$$
\mathbf{L}_{6}=\mathbf{A}_{6}-\mathbf{I}, \mathbf{L}_{18}=\mathbf{A}_{18}-\mathbf{I}, \mathbf{L}_{26}=\mathbf{A}_{26}-\mathbf{I}
$$

In the next sections, we express these operations as matrices and compute the spectral radii of these operations.

Setting $f_{k}\left(x_{1}, x_{2}, \cdots, x_{n}\right)$ to be the $k$ th-order fundamental symmetry forms of $\left\{x_{i}\right\}_{i=1}^{n}$, that is,

$$
f_{k}=\sum_{\text {all }}\left\{k \text { th-order mononimals of }\left\{x_{i}\right\}_{i=1}^{n}\right\},
$$

for example,

$$
\begin{aligned}
f_{1}\left(x_{1}, x_{2}, \cdots, x_{n}\right) & =x_{1}+x_{2}+\cdots+x_{n}, \\
f_{2}\left(x_{1}, x_{2}, \cdots, x_{n}\right) & =x_{1} x_{2}+x_{2} x_{3}+\cdots+x_{n-1} x_{n}, \\
f_{3}\left(x_{1}, x_{2}, \cdots x_{n}\right) & =x_{1} x_{2} x_{3}+x_{2} x_{3} x_{4}+\cdots+x_{n-2} x_{n-1} x_{n}, \\
& \vdots \\
f_{n}\left(x_{1}, x_{2}, \cdots, x_{n}\right) & =x_{1} x_{2} \cdots x_{n},
\end{aligned}
$$

the $k$ th-order fundamental symmetry forms $\left\{f_{k}\right\}_{i=1}^{n}$ satisfies the relation

$$
\Pi_{i=1}^{n}\left(1+x_{i}\right)=1+\sum_{k=1}^{n} f_{k}\left(x_{1}, x_{2}, \cdots, x_{n}\right) .
$$




\section{Operations in Matrix Form}

\subsection{Differential Operation in Matrix Form}

From the matrix

$$
\boldsymbol{D}=\left(\begin{array}{ccccccc}
-2 & 1 & 0 & 0 & \cdots & 0 & 0 \\
1 & -2 & 1 & 0 & \cdots & 0 & 0 \\
0 & 1 & 0 & 1 & \cdots & 0 & 0 \\
\vdots & \vdots & \vdots & \vdots & \ddots & \vdots & \vdots \\
0 & 0 & 0 & \cdots & 0 & 1 & -2
\end{array}\right)
$$

which computes the second-order derivative [10111315], we define the matrix $B$,

$$
\boldsymbol{B}=\frac{1}{2}\left(\begin{array}{ccccccc}
0 & 1 & 0 & 0 & \cdots & 0 & 0 \\
1 & 0 & 1 & 0 & \cdots & 0 & 0 \\
0 & 1 & 0 & 1 & \cdots & 0 & 0 \\
\vdots & \vdots & \vdots & \vdots & \ddots & \vdots & \vdots \\
0 & 0 & 0 & \cdots & 0 & 1 & 0
\end{array}\right)=\frac{1}{2}(\boldsymbol{D}+2 \boldsymbol{I})
$$

The matrix $\boldsymbol{B}$ computes the average of vectors. Setting $\rho(\boldsymbol{B})$ to be the spectrum of the matrix $\boldsymbol{B}$, we have the next theorem.

Theorem 1. The matrix $\boldsymbol{B}$ satisfies the relation $\rho(\boldsymbol{B})<1$. For the proof, see the appendix.

\subsection{Matrix Ring over the Kronecker Product and Matrix Addition}

Setting $\boldsymbol{A} \otimes \boldsymbol{B}$ to be the Kronecker product 14 15] of a pair of matrices, for the matrix $\boldsymbol{A}$, we define the symbol $\boldsymbol{A}^{\otimes n}$ as

$$
\boldsymbol{A}^{\otimes n}=\boldsymbol{A} \otimes \boldsymbol{A} \otimes \cdots \otimes \boldsymbol{A} .
$$

Over the collection of symmetry matrices, the Kronecker product $\otimes$ and the matrix sum + define the noncommutative $\operatorname{ring}[8,9] M(S, \otimes,+)$. Since the $n$ thorder fundamental symmetry forms $\left\{f_{k}(\boldsymbol{A})\right\}_{k=1}^{n}$ of the symmetry matrix $\boldsymbol{A}$ on $M(S, \otimes,+)$ is defined as

$$
f_{k}(\boldsymbol{A})=\sum_{\text {all }}\{\text { the Kronecker products of } k \boldsymbol{A} \text { and }(n-k) \boldsymbol{I}\}
$$

the symmetry forms are generated by

$$
(\boldsymbol{I}+\boldsymbol{A})^{\otimes n}=\boldsymbol{I}+\sum_{k=1}^{n} f_{k}(\boldsymbol{A})
$$

Since, for $\boldsymbol{A} \otimes \boldsymbol{A}$, the eigenvalue is $\lambda_{i} \lambda_{j}$, we have the following relation. 
Lemma 1. for the $n \times n$ regular symmetry matrix $\boldsymbol{A}$, setting $\left\{\lambda_{k}\right\}_{k=1}^{m}$ to be the eigenvalue of $\boldsymbol{A}$,

$$
f_{k}(\boldsymbol{A}) \boldsymbol{u}=f_{k}\left(\lambda_{1}, \lambda_{2}, \cdots, \lambda_{n}\right) \boldsymbol{u} .
$$

Since the number of terms of $f_{k}(\boldsymbol{A})$ is $\left(\begin{array}{l}n \\ k\end{array}\right)$, Lemma 1 implies the next theorem.

Theorem 2. The spectral radii of $f_{k}(\boldsymbol{A})$ satisfies the relation

$$
\rho\left(f_{k}(\boldsymbol{A})\right) \leq\left(\begin{array}{l}
n \\
k
\end{array}\right) \rho(\boldsymbol{A}) .
$$

\subsection{Averages in the Neighbourhood}

Using $\left\{f_{k}(\boldsymbol{A})\right\}_{k=1}^{n}$, we define the operations for the computation of the average in the neighbourhood and the Laplacian in the various neighbourhoods in $\mathbf{Z}^{n}$.

Setting

$$
N_{n(k)}(\boldsymbol{x})=\left\{\boldsymbol{x}^{\prime} \in \mathbf{Z}^{n},\left|\boldsymbol{x}-\boldsymbol{x}^{\prime}\right|=k\right\}
$$

we have the relation

$$
n(k)=\left|N_{n(k)}\right|=\left(\begin{array}{c}
n \\
k
\end{array}\right) 2^{k},
$$

since the number of terms in $f_{k}(\boldsymbol{B})$ is $\left(\begin{array}{l}n \\ k\end{array}\right)$.

Therefore, setting

$$
\boldsymbol{N}_{n(k)}=f_{k}(\boldsymbol{B})
$$

the matrix

$$
\boldsymbol{A}_{n(k)}=\left(\begin{array}{c}
n \\
k
\end{array}\right)^{-1} \boldsymbol{N}_{n(k)}
$$

computes the average of the points $\boldsymbol{x}^{\prime}$ in $N_{n(k)}(\boldsymbol{x})$. Since $\rho(\boldsymbol{B})<1$, eq. (7) implies the next lemma.

Lemma 2. Since $\rho\left(f_{k}\left(\boldsymbol{N}_{n(k)}\right)\right) \leq\left(\begin{array}{c}n \\ k\end{array}\right)$, we have the relation $\rho\left(f_{k}\left(\boldsymbol{A}_{n(k)}\right)\right) \leq 1$.

From the algebraic property of the fundamental symmetry forms, the matrices $\left\{\boldsymbol{N}_{n(k)}\right\}_{k=1}^{n}$ are generated from $(\boldsymbol{I}+\boldsymbol{B})^{\otimes n}$ as

$$
(\boldsymbol{I}+\boldsymbol{B})^{\otimes n}=\boldsymbol{I}+\sum_{k=1}^{n} \boldsymbol{N}_{n(k)}
$$

We define the matrix of the Laplacian with $\boldsymbol{x}$ and $\boldsymbol{x}^{\prime} \in N_{d(k)}$ as the relation

$$
\boldsymbol{L}_{d(k)}=f_{k}(\boldsymbol{D}) \text {. }
$$

In particular, $\boldsymbol{L}_{n(1)}$ is the discrete Laplacian with $2 n$ connectivity in $\mathbf{Z}^{n}$, that is,

$$
\boldsymbol{L}_{n(1)}=\boldsymbol{D} \otimes \boldsymbol{I} \otimes \cdots \otimes \boldsymbol{I}+\boldsymbol{I} \otimes \boldsymbol{D} \otimes \cdots \otimes \boldsymbol{I}+\cdots+\boldsymbol{I} \otimes \boldsymbol{I} \otimes \cdots \otimes \boldsymbol{D} .
$$

Similarly to eq. (12), we have the relation

$$
(\boldsymbol{I}+\boldsymbol{D})^{\otimes n}=\boldsymbol{I}+\sum_{k=1}^{n} \boldsymbol{L}_{d(k)} .
$$




\subsection{Construction of Laplacian Matrices}

We define the $r$ th Laplacian in $\mathbf{Z}^{n}$ in the matrix form as

$$
\boldsymbol{L}_{n(k)}=\boldsymbol{A}_{n(k)}-\boldsymbol{I}
$$

As the generalisation of the Horn-Schunck Laplacian [16] in $\mathbf{Z}^{n}$, we have

$$
\boldsymbol{L}_{(m)}=\sum_{i=k}^{m} \alpha_{k} \boldsymbol{A}_{n(k)}-\boldsymbol{I}, \quad 1 \leq m \leq n
$$

for

$$
\alpha_{1}: \alpha_{2}: \cdots: \alpha_{n}=n:(n-1): \cdots: 1 .
$$

\section{$3.5\left(m^{n}-1\right)$ Neighbourhood Operation}

We define the matrix $\boldsymbol{B}^{(k)}=\left(\left(b_{i j}\right)\right)$ as

$$
b_{i j}= \begin{cases}\frac{1}{2} \delta_{|i-j| 0}, & i \neq j, \\ 1 & \text { otherwise. }\end{cases}
$$

Then, we have the relations $\boldsymbol{B}^{(1)}=\boldsymbol{B}$ and $\boldsymbol{B}^{(0)}=\boldsymbol{I}$.

Setting

$$
N_{+}^{m}=\left\{\boldsymbol{x}=(i(1), i(2), \cdots, i(n))^{\top} \mid 0 \leq i(1), i(2), \cdots i(n) \leq \frac{m+1}{2}\right\},
$$

we define the matrix

$$
\boldsymbol{B}^{(i(1) i(2) \cdots i(n))}=\boldsymbol{B}^{(i(1))} \otimes \boldsymbol{B}^{(i(2))} \otimes \cdots \otimes \boldsymbol{B}^{(i(n))} .
$$

Matrix $\boldsymbol{B}^{(i(1) i(2) \cdots i(n))}$ computes the average of the points $\boldsymbol{p}=(\mid i(1))|| i,(2) \mid, \cdots$, $|i(n)|)^{\top}$ in $\mathbf{Z}^{n}$. Therefore, the matrix

$$
\boldsymbol{N}_{n}=\sum_{(i(1) i(2) \cdots i(n)) \in N_{+}^{n}} \gamma_{(i(1) i(2) \cdots i(n))} \boldsymbol{B}^{(i(1) i(2) \cdots i(n))}-\gamma_{00 \cdots 0} \boldsymbol{I}
$$

for

$$
\sum_{(i(1) i(2) \cdots i(n)) \in N_{+}^{n}} \gamma_{(i(1) i(2) \cdots i(n))}=1,0<\gamma_{(i(1) i(2) \cdots i(n))}<1
$$

computes the weighted average of $f_{\boldsymbol{p}}, \boldsymbol{p} \in \mathbf{Z}^{n}$ for the points in the $\left(m^{n}-1\right)$ neighbourhood of each point. For $\rho\left(\boldsymbol{B}^{(i(1) i(2) \cdots i(n))}\right) \leq 1$, we have the property that $\rho\left(\boldsymbol{N}_{n}\right) \leq 1$.

\section{Operations in $\mathrm{Z}^{3}$}

In this section, we construct the Laplacian and average operation in $\mathbf{Z}^{3}$ using the results in the previous section. 
6-Neighbourhood Operations. Using the matrix $\boldsymbol{B}$, we can construct the operation to compute the average of digital functions in the 6-neighbourhood.

Theorem 3. The matrix

$$
\boldsymbol{A}_{6}=\frac{1}{3}(\boldsymbol{B} \otimes \boldsymbol{I} \otimes \boldsymbol{I}+\boldsymbol{I} \otimes \boldsymbol{B} \otimes \boldsymbol{I}+\boldsymbol{I} \otimes \boldsymbol{I} \otimes \boldsymbol{B})
$$

computes the average of $f_{i j k}$ in the 6-neighbourhood of the point $(i, j, k)^{\top}$.

The matrix of the 6-neighbourhood Laplacian is

$$
\boldsymbol{L}_{6}=\frac{1}{6}(\boldsymbol{D} \otimes \boldsymbol{I} \otimes \boldsymbol{I}+\boldsymbol{I} \otimes \boldsymbol{D} \otimes \boldsymbol{I}+\boldsymbol{I} \otimes \boldsymbol{I} \otimes \boldsymbol{D}) .
$$

18-Neighbourhood Operations. The simple Laplacian in $\mathbf{Z}^{2}$ is

$$
\boldsymbol{L}_{8}=\boldsymbol{L}_{4}+\frac{1}{4}(\boldsymbol{D} \otimes \boldsymbol{D}) .
$$

Equation (25) shows that the 8-neighbourhood Laplacian on $\mathbf{Z}^{2}$ involves the fourth-order differentiation for the points $(i-1, j-1)^{\top},(i+1, j+1)^{\top},(i+$ $1, j-1)^{\top}$, and $(i+1, j+1)^{\top}$. Therefore, from the viewpoint of the numerical computation, it is desired to use the Laplacian in the form

$$
\boldsymbol{L}_{8}=\alpha_{1} \boldsymbol{L}_{4}+\alpha_{2} \frac{1}{4}(\boldsymbol{D} \otimes \boldsymbol{D}) .
$$

such that $\alpha_{1} \gg \alpha_{2}>0$.

Equation (17) derives the next relation for $n=2$,

$$
\boldsymbol{L}_{8 H S}=\frac{2}{3} \boldsymbol{L}_{4}+\frac{1}{3}\left\{\frac{1}{4}(\boldsymbol{D} \otimes \boldsymbol{D})\right\} .
$$

This matrix is expressed in the $3 \times 3$ tensor as

$$
\mathbf{L}_{8 H S}=\frac{2}{3}\left(\begin{array}{ccc}
0 & 1 & 0 \\
1 & -4 & 1 \\
0 & 1 & 0
\end{array}\right)+\frac{1}{3}\left(\begin{array}{ccc}
1 & 0 & 1 \\
0 & -4 & 0 \\
1 & 0 & 1
\end{array}\right)=\frac{1}{12}\left(\begin{array}{ccc}
1 & 2 & 1 \\
2 & -12 & 2 \\
1 & 2 & 1
\end{array}\right) .
$$

In this section, we clarify the three-dimensional analogue of the operation of eq. (27).

For the matrix

$$
\boldsymbol{A}_{12}=\frac{1}{3}(\boldsymbol{B} \otimes \boldsymbol{B} \otimes \boldsymbol{I}+\boldsymbol{B} \otimes \boldsymbol{B} \otimes \boldsymbol{I}+\boldsymbol{B} \otimes \boldsymbol{I} \otimes \boldsymbol{B}),
$$

we have the next theorem.

Theorem 4. The matrix $\boldsymbol{B}_{18}$, such that

$$
\boldsymbol{B}_{18}=\frac{6}{18} \boldsymbol{B}_{6}+\frac{12}{18} \boldsymbol{B}_{12},
$$

computes the average in the 18-neighbourhood of each point. 
From this theorem, the Laplacian in the 18-neighbourhood is given as

$$
\begin{aligned}
\boldsymbol{L}_{18} & =\boldsymbol{B}_{18}-\boldsymbol{I} \\
& =\frac{6}{18} \boldsymbol{B}_{6}+\frac{12}{18} \boldsymbol{B}_{12} \\
& =\frac{6}{18}\left(\boldsymbol{B}_{6}-\boldsymbol{I}\right)+\frac{12}{18}\left(\boldsymbol{B}_{12}-\boldsymbol{I}\right) \\
& =\frac{1}{3} \boldsymbol{L}_{6}+\frac{1}{3}\{(\boldsymbol{B} \otimes \boldsymbol{B} \otimes \boldsymbol{I}+\boldsymbol{I} \otimes \boldsymbol{B} \otimes \boldsymbol{B}+\boldsymbol{B} \otimes \boldsymbol{I} \otimes \boldsymbol{B}-\boldsymbol{I}\} \\
& =\frac{1}{3} \boldsymbol{L}_{6}+\frac{2}{3}\{\boldsymbol{D} \otimes \boldsymbol{D} \otimes \boldsymbol{I}+\boldsymbol{I} \otimes \boldsymbol{D} \otimes \boldsymbol{D}+\boldsymbol{D} \otimes \boldsymbol{I} \otimes \boldsymbol{D}\} .
\end{aligned}
$$

The second term of the right-hand side of eq. (31) is the fourth-order numerical differentiation, since $\boldsymbol{D} \otimes \boldsymbol{D}$ corresponds to the fourth time derivatives. This algebraic property implies the next assertion.

Assertion 1. The 18-neighbourhood Laplacian $\mathbf{L}$ is a sum of the 6neighbourhood Laplacian and a fourth-order symmetry differentiation.

The three-dimensional version of the Horn-Schunck Laplacian is expressed in the $3 \times 3 \times 3$ tensor form as

$$
\mathbf{L}_{H S}=\left(\frac{1}{36}\left(\begin{array}{lll}
0 & 1 & 0 \\
1 & 4 & 1 \\
0 & 1 & 0
\end{array}\right) \frac{1}{36}\left(\begin{array}{ccc}
1 & 4 & 1 \\
4 & -36 & 4 \\
1 & 4 & 1
\end{array}\right) \frac{1}{36}\left(\begin{array}{lll}
0 & 1 & 0 \\
1 & 4 & 1 \\
0 & 1 & 0
\end{array}\right)\right)
$$

where the elements express the weights for the computation of the Laplacian of the centre of the tensor, that is, $\mathbf{L}$ is the weighted 18-neighbourhood operation. This operation is decomposed into two tensors as

$$
\begin{aligned}
\mathbf{L}_{H S}= & \frac{2}{3}\left(\left(\begin{array}{lll}
0 & 0 & 0 \\
0 & 1 & 0 \\
0 & 0 & 0
\end{array}\right)\left(\begin{array}{ccc}
0 & 1 & 0 \\
1 & -6 & 1 \\
0 & 1 & 0
\end{array}\right)\left(\begin{array}{lll}
0 & 0 & 0 \\
0 & 1 & 0 \\
0 & 0 & 0
\end{array}\right)\right) \\
& +\frac{1}{3}\left(\left(\begin{array}{lll}
0 & 1 & 0 \\
1 & 0 & 1 \\
0 & 1 & 0
\end{array}\right)\left(\begin{array}{ccc}
1 & 0 & 1 \\
0 & -12 & 0 \\
1 & 0 & 1
\end{array}\right)\left(\begin{array}{lll}
0 & 1 & 0 \\
1 & 0 & 1 \\
0 & 1 & 0
\end{array}\right)\right) .
\end{aligned}
$$

The first term and the second term of the right-hand side of eq. (33) are the 6neighbourhood Laplacian and the 12-neighbourhood Laplacian, respectively, in three-dimensional discrete space. Therefore, the three-dimensional Horn-Schunck Laplacian is in the form

$$
\begin{aligned}
\boldsymbol{L}_{H S}= & \frac{2}{3} \boldsymbol{L}_{6}+\frac{1}{3} \boldsymbol{L}_{18} \\
= & \frac{2}{3}(\boldsymbol{D} \otimes \boldsymbol{I} \otimes \boldsymbol{I}+\boldsymbol{I} \otimes \boldsymbol{D} \otimes \boldsymbol{I}+\boldsymbol{I} \otimes \boldsymbol{I} \otimes \boldsymbol{D}) \\
& +\frac{1}{3}(\boldsymbol{D} \otimes \boldsymbol{D} \otimes \boldsymbol{I}+\boldsymbol{D} \otimes \boldsymbol{I} \otimes \boldsymbol{D}+\boldsymbol{I} \otimes \boldsymbol{D} \otimes \boldsymbol{D}) .
\end{aligned}
$$


26-Neighbourhood Operations. In digital image analysis, the operations in the 26-neighbourhood are used, since the operations in the 26-neighbourhood derive the smoother results than the 6- and 18- neighbourhood operations. In this section, we examine the norm properties of the operations in the 26neighbourhood. For the matrices $\boldsymbol{A}_{6}, \boldsymbol{A}_{12}$, and

$$
\boldsymbol{A}_{8}=\boldsymbol{B} \otimes \boldsymbol{B} \otimes \boldsymbol{B}
$$

we have the next theorem.

Theorem 5. The matrix $\boldsymbol{B}_{26}$, such that

$$
\boldsymbol{B}_{26}=\frac{6}{26} \boldsymbol{A}_{6}+\frac{12}{26} \boldsymbol{A}_{12}+\frac{8}{26} \boldsymbol{A}_{8}
$$

computes the averages in the 26-neighbourhood of each point.

From this theorem, we can have the Laplacian in the 18-neighbourhood as

$$
\begin{aligned}
\boldsymbol{L}_{26} & =\boldsymbol{B}_{26}-\boldsymbol{I} \\
& =\frac{6}{26} \boldsymbol{L}_{6}+\frac{12}{26} \boldsymbol{L}_{12}+\frac{8}{26} \boldsymbol{L}_{8}
\end{aligned}
$$

As the 26-neighbourhood Horn-Schunck Laplacian is given as

$$
\boldsymbol{L}_{26 H S}=\frac{3}{6} \boldsymbol{L}_{6}+\frac{2}{6} \boldsymbol{L}_{12}+\frac{1}{6} \boldsymbol{L}_{8}=\frac{1}{2} \boldsymbol{L}_{6}+\frac{1}{3} \boldsymbol{L}_{12}+\frac{1}{6} \boldsymbol{L}_{8} .
$$

\subsection{Discrete Gaussian Kernel}

By embedding signal sequences as

$$
\boldsymbol{f}=\left(f_{1}, f_{2}, \cdots, f_{n}\right)^{\top} \rightarrow\left(0,0, \cdots, f_{1}, f_{2}, \cdots, f_{n}, 0, \cdots, 0\right)^{\top}
$$

with an appropriate number of zeros, a discrete version of the heat equation

$$
\frac{\partial}{\partial t} f(x, t)=\frac{1}{2} \frac{\partial^{2}}{\partial x^{2}} f(x, t)
$$

is given as

$$
\boldsymbol{f}^{(t+1)}-\boldsymbol{f}^{(t)}=\frac{1}{4} \boldsymbol{D} \boldsymbol{f}^{(t)}, \boldsymbol{f}^{(0)}=\boldsymbol{f} .
$$

From this equation, we have the relation

$$
\boldsymbol{f}^{(t+1)}=\boldsymbol{G} \boldsymbol{f}^{(t)}=\left(\boldsymbol{I}+\frac{1}{4} \boldsymbol{D}\right) \boldsymbol{f}^{(t)}=\frac{1}{2}(\boldsymbol{I}+\boldsymbol{B}) \boldsymbol{f}^{(t)}=\boldsymbol{G}^{t} \boldsymbol{f}
$$

where

$$
\boldsymbol{G}=\frac{1}{4}\left(\begin{array}{ccccc}
2 & 1 & 0 & \cdots & 0 \\
1 & 2 & 1 & \cdots & 0 \\
\vdots & 1 & 2 & \cdots & 0 \\
\vdots & \vdots & \vdots & \ddots & \vdots \\
0 & \cdots & \cdots & 1 & 2
\end{array}\right)
$$


The $n \mathrm{D}$ kernel is expressed as

$$
\boldsymbol{G}_{n}^{t}=\left(\boldsymbol{G}^{\otimes n}\right)^{t}=(\boldsymbol{G} \otimes \boldsymbol{G} \otimes \cdots \otimes \boldsymbol{G})^{t}=\left(\boldsymbol{G}^{t} \otimes \boldsymbol{G}^{t} \otimes \cdots \otimes \boldsymbol{G}^{t}\right)
$$

Since the 1D Gaussian convolution sequence is $\boldsymbol{g}=\left(\frac{1}{4}, \frac{1}{2}, \frac{1}{4}\right)^{\top}$, the 3D kernel in the tensor form is $\mathbf{G}_{3}^{t}$ for $\mathbf{G}=\left(\mathbf{G}_{-1}, \mathbf{G}_{0}, \mathbf{G}_{1}\right)$ where

$$
\boldsymbol{G}_{-1}=\frac{1}{4}\left(\begin{array}{ccc}
\frac{1}{16} & \frac{1}{8} & \frac{1}{16} \\
\frac{1}{8} & \frac{1}{4} & \frac{1}{8} \\
\frac{1}{16} & \frac{1}{8} & \frac{1}{16}
\end{array}\right), \quad \boldsymbol{G}_{0}=\frac{1}{2}\left(\begin{array}{ccc}
\frac{1}{16} & \frac{1}{8} & \frac{1}{16} \\
\frac{1}{8} & \frac{1}{4} & \frac{1}{8} \\
\frac{1}{16} & \frac{1}{8} & \frac{1}{16}
\end{array}\right), \quad \boldsymbol{G}_{+1}=\frac{1}{4}\left(\begin{array}{ccc}
\frac{1}{16} & \frac{1}{8} & \frac{1}{16} \\
\frac{1}{8} & \frac{1}{4} & \frac{1}{8} \\
\frac{1}{16} & \frac{1}{8} & \frac{1}{16}
\end{array}\right) \text {. }
$$

\section{Conjectures for the Neighbourhood in $\mathrm{Z}^{n}$}

In four-dimensional discrete space $\mathbf{Z}^{4}$, elemental operations, which is derived as the fundamental symmetry forms of $\boldsymbol{B}$ on $M(S, \otimes,+)$, for the computation of the averages in the neighbourhoods are

$$
\begin{aligned}
\boldsymbol{A}_{8}= & \frac{1}{4}(\boldsymbol{B} \otimes \boldsymbol{I} \otimes \boldsymbol{I} \otimes \boldsymbol{I}+\boldsymbol{I} \otimes \boldsymbol{B} \otimes \boldsymbol{I} \otimes \boldsymbol{I} \\
& +\boldsymbol{I} \otimes \boldsymbol{I} \otimes B \otimes \boldsymbol{I}+\boldsymbol{I} \otimes \boldsymbol{I} \otimes \boldsymbol{I} \otimes \boldsymbol{B}), \\
\boldsymbol{A}_{24}= & \frac{1}{6}(\boldsymbol{B} \otimes \boldsymbol{B} \otimes \boldsymbol{I} \otimes \boldsymbol{I}+\boldsymbol{I} \otimes \boldsymbol{B} \otimes \boldsymbol{B} \otimes \boldsymbol{I}+\boldsymbol{I} \otimes \boldsymbol{I} \otimes B \otimes \boldsymbol{B} \\
& +\boldsymbol{B} \otimes \boldsymbol{I} \otimes b m I \otimes \boldsymbol{B}+\boldsymbol{B} \otimes \boldsymbol{I} \otimes \boldsymbol{B} \otimes \boldsymbol{I}+\boldsymbol{I} \otimes \boldsymbol{B} \otimes \boldsymbol{I} \otimes \boldsymbol{B}), \\
\boldsymbol{A}_{32}= & \frac{1}{4}(\boldsymbol{B} \otimes \boldsymbol{B} \otimes \boldsymbol{B} \otimes \boldsymbol{I}+\boldsymbol{I} \otimes \boldsymbol{B} \otimes \boldsymbol{B} \otimes \boldsymbol{B} \\
& +\boldsymbol{B} \otimes \boldsymbol{I} \otimes \boldsymbol{B} \otimes \boldsymbol{B}+\boldsymbol{I} \otimes \boldsymbol{B} \otimes \boldsymbol{I} \otimes \boldsymbol{B}), \\
\boldsymbol{A}_{16}= & \boldsymbol{B} \otimes \boldsymbol{B} \otimes \boldsymbol{B} \otimes \boldsymbol{B} .
\end{aligned}
$$

In three-dimensional discrete space, $\boldsymbol{A}_{12}$ and $\frac{6}{14} \boldsymbol{A}_{6}+\frac{8}{14} \boldsymbol{A}_{8}$ correspond to the averages in the FCC neighbourhood and the BCC neighbourhood, respectively. From these properties of the operations in the noncubic neighbourhoods, we have the following open problem and conjecture.

Open Problem 1. Using the fundamental symmetry forms of $\boldsymbol{B}$ on $M(S, \otimes$, $+)$, characterise the noncubic grid systems in $\mathbf{Z}^{n}$.

In $\mathbf{Z}^{4}, \boldsymbol{A}_{12}$ and $\left\langle\boldsymbol{A}_{6}, \boldsymbol{A}_{8}\right\rangle$ define the neighbourhood point configurations for FCC and BCC grids [2], respectively. From these geometric properties of the noncubic grids embedded in $\mathbf{Z}^{4}$, we have the next conjecture for the noncubic grids.

Conjecture 1. The pairs $\left\langle\boldsymbol{A}_{n(k)}, \boldsymbol{A}_{n(n-k)}\right\rangle$ for $k=1,2, \cdots,(n-1)$ derive the $n$-dimensional versions of FCC and BCC grids.

\section{Conclusions}

We introduced a decomposition and construction method for the neighbourhood operations of the digital image processing. First, we have derived a method 
to express the symmetry neighbourhood operations as matrices. The matrix expressions of the neighbourhood operations derived the operator norm of the operations. This norm of the neighbourhood operations allowed us to deal with the neighbourhood operations analytically, that is, we defined the spectra of the neighbourhood operations.

\section{References}

1. Klette, R., Rosenfeld, A.: Digital Geometry: Geometric Method for Digital Picture Analysis. Morgan Kaufmann, San Francisco (2004)

2. Serra, J.: Mathematical Morphology. Academic Press, London (1982)

3. Bracewell, R.N.: Two Dimensional Imaging. Prentice-Hall, Englewood Cliffs (1995)

4. Bellman, R.: Introduction to Matrix Analysis, 2nd edn. SIAM, Philadelphia (1987)

5. Strang, G., Nguyen, T.: Wavelets and Filter Banks. Wellesley-Cambridge (1997)

6. Fuhrmann, P.A.: Polynomial Approach to Linear Algebra. Springer, Heidelberg (1996)

7. Mignotte, M., Stefanescu, D.: Polynomials: An Algorithmic Approach. Springer, Heidelberg (1999)

8. Clausen, M., Baum, J.: Fast Fourier Transform. Bibliographishes Institut \& F.A, Brockhaus (1993)

9. Terras, A.: Fourier Analysis on Finite Groups and Applications, Cambridge (1999)

10. Demmel, J.W.: Applied Numerical Linear Algebra. SIAM, Philadelphia (1997)

11. Grossmann, C., Roos, H.-G.: Numerik partieller Differentialgleichungen, Teubner (1994)

12. Grossmann, C., Terno, J.: Numerik der Optimierung, Teubner (1997)

13. Varga, R.S.: Matrix Iteration Analysis, 2nd edn. Springer, Heidelberg (2000)

14. Graham, A.: Kronecker Products and matrix Calculus with Applications. Ellis Horwood (1981)

15. Ruíz-Tolosa, J.R., Castillo, E.: From Vectors to Tensors. Springer, Heidelberg (2005)

16. Horn, B.K.P., Schunck, B.G.: Determining optical flow. Artificial Intelligence 17, 185-204 (1981)

\section{Appendix}

\section{Spectrums of Matrices}

For the matrix $\boldsymbol{D}$, setting $\boldsymbol{D} \boldsymbol{U}=\boldsymbol{\Lambda} \boldsymbol{U}$, where $\boldsymbol{U}$ is an orthogonal matrix, and $\boldsymbol{\Lambda}=\operatorname{Diag}\left(\lambda_{M}, \lambda_{M-1}, \cdots, \lambda_{1}\right)$, the eigenvalues are $\lambda_{k}=-2\left(1-\cos \frac{\pi}{M+1} k,\right)$. Since $\boldsymbol{B}=\frac{1}{2}(\boldsymbol{D}+2 \boldsymbol{I})$, we have the eigenvalues of $\boldsymbol{B}$ as $\mu_{k}=\cos \frac{\pi}{M+1} k$. Therefore, $\rho(\boldsymbol{B})<1$. Using the same treatment on the fundamental symmetrical polynomial of $\mu_{i}$, we can have the relation $\rho\left(\boldsymbol{A}_{n(r)}\right)<1$. Furthermore, since $\rho\left(\boldsymbol{B}^{n(r)}\right) \leq$ $\max _{i} \sum_{j}\left|b_{i j}^{n(r)}\right|=1$, we have $\rho\left(\boldsymbol{B}^{(i)}\right) \leq 1$. 